\title{
Energy efficiency optimization-based resource allocation for underlay RF-CRN with residual energy and QoS guarantee
}

\author{
Jie Tian ${ }^{1}, \mathrm{He}_{\text {Xiao }}^{2}$, Yimao Sun ${ }^{1}$, Dong Hou ${ }^{3}$ and Xianglu Li ${ }^{1 *}$
}

\author{
*Correspondence: bluelx|@163.com \\ ${ }^{1}$ Institute of Electronic Engineering, \\ China Academey of Engineering \\ Physics, No. 64 Mianshan Road, \\ 621900 Mianyang, China \\ Full list of author information is \\ available at the end of the article
}

\begin{abstract}
How to achieve energy-efficient transmission in radio frequency energy harvesting cognitive radio network (RF-CRN) is of great importance when nodes in CRN are self-maintained. This paper presents a radio frequency (RF) energy harvesting hardware-based underlay cognitive radio network (RH-CRN) structure, where a secondary transmitter (ST) first harvests energy from RF signals source originating from the primary network, and then communicates with a secondary receiver (SR) in underlay mode by using the harvested energy. The total consumed energy by the secondary user (SU) must be equal to or less than the total harvested energy referred to as energy causality constraint, In addition, the ST possesses some initial energy which may be the residual energy from the former transmission blocks, and we consider the energy loss of energy harvesting circuit as a systematic factor as well. Our goal is to achieve the maximum energy efficiency (EE) of the secondary network by jointly optimizing transmitting time and power. To guarantee the quality of service (QoS) of secondary transceiver, a minimum requirement of throughput constraint is imposed on the ST in the process of EE maximization. As the EE maximization is a nonlinear fractional programming problem, a quick iterative algorithm based on Dinkelbach's method is proposed to achieve the optimal resource allocation. Simulation results show that the proposed strategy has fast convergence and can improve the system EE greatly while ensuring the QoS.

Keywords: Energy harvesting, Cognitive radio network, Energy efficiency, Residual energy, QoS, Dinkelbach, Resource allocation
\end{abstract}

\section{Introduction}

Radio frequency (RF) energy harvesting-based (EH) cognitive radio network (RF-CRN), which has emerged as a promising way to address the problems of spectrum scarcity and energy efficiency while consistent with the call for green communication at the same time, has received extensive attention over the recent years [1]. RF energy harvesting technique allows wireless nodes to collect energy of electromagnetic waves sent from RF sources (e.g., TV, radio towers and cellular base-stations) and then convert it to electrical energy, which can be utilized for data transmission and self-powering. Comparing to the 
traditional EH sources, such as solar, wind, wave, and heat, the RF energy harvesting is more flexible and sustainable since the RF signals radiated by ambient transmitters could be consistently available and has little limitations on time, space, or locations [2,3].

RF energy sources can be mainly categorized into dedicated RF sources and ambient RF sources. Dedicated RF sources can power the wireless nodes which require stable and predictable energy due to on-demand power supply and directional transmission. For example, wireless powered communication networks (WPCN) in which distributed wireless devices are powered via dedicated wireless energy transfer (WET) by the hybrid access point (H-AP) in the downlink (DL) for wireless information transmission (WIT) in the uplink (UL) [4-9], unlike previous studies on simultaneous wireless information and power transfer (SWIPT) in which the wireless information and energy are included in downlink RF signals at the same time [10-12].

In the scenarios of $\mathrm{CRN}$, ambient RF signals contain most of the radiations from nearby RF sources such as base stations, primary licensed networks, and other secondary sources. It is not dedicated for EH but freely available, can be used in self-powered scenarios where its hard to change batteries or recharge frequently for low-power devices, such as wireless sensor networks (WSNs). The RF signals radiated by the primary networks are not only interference for the secondary users (SUs), but also can be regarded as the green energy sources for $\mathrm{EH}$, so that SUs can utilize both spectrum and energy of primary networks during the working process.

Based on the approaches of collecting the ambient RF energy and access the licensed spectrum, SUs in RF-CRN mainly operate in three typical modes, namely, interweave, overlay, and underlay [13]. In interweave mode, SUs first harvest RF energy, and then spend the energy on spectrum sensing and opportunistically access the licensed spectrum for transmitting wireless information when primary users (PUs) are detected as inactive. With the assumption that SUs have prior knowledge of PU's transmitting sequence and encoding scheme, the harvested energy is used to serve the transmission of both PU and SU in overlay mode [3, 14-17]. Unlike the sensing energy consumption and discontinuous data transmission in interweave mode and unnecessary collaboration of PUs and SUs in overlay mode, SU should transmit along with PU as long as the interference caused by the secondary transmission at primary receiver (PR) keeps below an acceptable peak threshold in underlay mode, so the important detection and false alarm probabilities in overlay mode need not be taken into consideration in underlay [13, 18-20].

For the sake of designing green communication networks, the SU nodes working in RFCRN are usually self-maintained by harvested environmental RF energy supplies other than dedicated power supplies. However, the harvested energy supplies is somewhat dynamic or unstable due to the transmitting power fluctuation of ambient RF sources or unpredictable channel fading. Therefore, it is necessary to maximize the energy efficiency (EE) in RF-CRN, which is defined as the ratio of the amount of average transmitted data bits to the amount of consumed energy [21]. As an important indicator in energyefficient for RF-CRN communications, tremendous works have been done in explicitly solving the EE maximization problem, which is a nonlinear fractional optimization problem [22] under the energy and interference constraints. Energy constraint requires that the total energy consumed by SUs must be equal to or less than the total harvested energy, and interference constraint only enables the transmitting power of SUs must be restricted to protect PUs from secondary interference. At the same time, throughput plays an 
important role in EE maximization for RF-CRNs. However, larger throughput threshold means more energy consumption; it is essential to minimize throughput that meets the QoS requirement of low priority users, so that they can harvest enough energy when primary users transmit high priority data continuously in data gathering, event monitoring, and other multi-priority WSN applications.

In this paper, we consider an underlay ambient RF-CRN system in which a battery-free SU first harvests energy from RF signal radiated by PU, and then transmits data by using harvested energy. Our goal is achieving the maximum of EE optimization in RF-CRN while guaranteeing the QoS requirement of SU, without affecting transmission of PU. We summarize three contributions in this paper.

- First, we formulate a novel EE maximization model of underlay RF-CRN as a nonlinear fractional programming problem under certain constraints, which is different from the works that design the paradigm to enhance SE of underlay RF-CRNs. Specifically, we take advantages of the residual energy after former transmission block, which enables more flexibility to energy utilization of SUs and generalizes the application scenario.

- Second, to ensure the QoS of SU, taking only energy and interference constraints into consideration for optimizing EE is not enough. We impose a minimum throughput requirement and residual energy of SU into the constraint conditions, and study the EE maximization problem with respect to joint allocation of harvesting time and transmitting power.

- Third, in order to solve the EE maximization which is a nonlinear fractional optimization problem, an alternative energy-efficient resource allocation algorithm based on Dinkelbach method and Lagrangian Dual model is proposed to obtain the optimal solution, with the features of fast convergence and insensitivity to the initial values of residual energy.

The rest of this paper is organized as follows. Section 2 reviews the related works on RF-CRNs. Section 3 introduces the system model and the problem formulation for the proposed RF-CRN. Section 4 presents the theoretical proof and solutions of the EE optimization. Section 5 analyzes the convergence and simulation result of the proposed algorithm. Section 6 concludes the paper and discusses the future work.

\section{Related works and motivation}

In order to maximize $\mathrm{EE}$ in $\mathrm{CRN}$, previous research works of energy reduction have been done in CRN.

Feng and Gan [23] presents the EE maximization, in which the dynamically sensing and transmission are formulated as a partially observable Markov decision process and resolved by myopic policy. By joint control of multi-channel allocation and transmitting power, EE is formulated as a nonlinear integer programming and solved by a polynomial time heuristic algorithm [24]. In [25], EE is formulated as a multi-objective optimization problem with respect to ergodic capacity and is solved by being transferred into a single objective problem with constraints. Furthermore, [26] proposes a general framework to evaluate the tradeoff between EE and spectrum efficiency (SE) in CRNs. Recently, [27] models the average EE maximization problem as a joint optimization of spectrum sensing duration and ST transmit power under the constraints of outage 
probability of SUs transmissions. However, the above works focus only on the energy efficiency of CRNs without considering EH technique, which may not be applicable on many occasions, such as wireless nodes that cannot replace batteries or recharge frequently in WSNs.

As a typical example of RF energy harvesting networks and a promising solution to achieve perpetually operating wireless networks, WPCN technique has been investigated in recent studies [4-9]. In [4], the authors consider an WPCN that nodes can save energy for later blocks, the sum rate can be maximized by optimally allocating time and energy without considering energy efficiency. Chen et al. [5] presents a wireless powering cooperative communication networks consisting of a hybrid access point(AP), a source, and a relay. The source and relay harvest energy from broadcasted signals for the cooperative transmission. In order to solve the near-far problem of EH efficiency due to UE random locations, authors in [6] propose an adaptive harvest-cooperate protocol; every UE harvests the emitted energy then sends data to AP directly or via other UEs acting as relays in a time division multiplexing (TDM) manner. The authors In [7] investigate the time and power allocation for DL WET and UL WIT to maximize EE of WPCN systems. In [8, 9], the authors present an iterative resource allocation based on the Dinkelbach structure and particle-swarm optimization algorithm to optimize $\mathrm{EE}$ as a nonlinear fractional programming problem. Since the RF signals can convey information and energy simultaneously, various models for different SWIPT applications have been studied to characterize the trade-offs between WET and WIT, including broadcast channels [10], interference [11], and relays [12].

However, the works on WPCNs or SWIPTs mentioned above are based on the assumption that the system can operate in exclusive frequency band, so there is no need to consider the same frequency interference caused by the other wireless systems. Recently, as a new type of CR enabled secondary WPCN, cognitive WPCN which shares spectrum with the primary system is presented [28]. In [29], a wireless powered underlay CRN where SUs first harvest energy in the DL wireless power transfer (WPT) phase and then use the energy in the TDM UL wireless information transmission phase is introduced. These works optimize SE for cognitive WPCN, so they mainly focus on maximizing the sum rate of the SU system under different constraints without considering energy efficiency.

Lastly, the literatures of ambient RF energy harvesting mainly focus on RF-CRN systems, in which SUs first harvest energy from PUs signals, and then use the harvested energy on data transmission by sharing spectrum with PUs. While keeping PUs sufficiently protected, [30] focuses on the harvesting-sensing-throughput tradeoff and jointly optimizes efficiency ratio, sensing duration, sensing threshold, and fusion rule to maximize SUs expected achievable throughput. In [31], SUs are able to not only transmit packets on an idle licensed channel, but also harvest RF energy from PUs transmitting signals when the channel is occupied. Wu et al. [32] considers the EE maximization of interweave RF-CRN by jointly optimizing sensing time and transmission power. A CDMA-based underlay CRN with RF energy harvesting is presented in [33]. Unlike the majority of previous RF-CRN studies, which consider CRNs under a single user setting, the goal of [15] is investigating the joint impact of sensing probability, access probability, and energy queue capacity on the maximum achievable throughput in a multiuser EH-CRN. In [34], a two-hop underlay cognitive relay network with an EH 
relay is proposed. Authors in [35] maximizes the EE of the SU network in which the ST needs to dynamically adjust the transmitting power according to the sensing results of the PU's state. When PUs stay active during most of the system's working time, SUs can hardly sense spectral holes for data transmission and there will be additional energy and time consumption on spectrum sensing and collaborative transmission for SUs respectively in interweave and overlay RF-CRN. Then, SUs can hardly have energy and time to transmit their own information, even if the throughput requirement is fairly low. So, the underlay RF-CRN framework can be proven to be potentially more energy-efficient in many practical scenarios [19]. Recently, an underlay multi-hop RFCRN is analyzed in [13], in which all the SUs harvest energy from PU signals to be self-powered.

These aforementioned works about RF-CRNs mainly focus on the fundamental tradeoff between achievable throughput and harvested energy. Specifically, by jointly optimizing the harvesting time and transmitting powers of SUs, the aim is to maximize the sum rate of the network, subject to the primary interference constraint and the energy causality constraint. Unfortunately, the energy harvesting efficiency of the nodes in small factor is usually low due to the design complexity of hardware. Achieving energy-efficient transmissions for ambient RF-CRNs is important, so that they can avoid excessive energy consumption and system outage [1]. To our best knowledge, the EE maximization with QoS guarantee in underlay RF-CRN is still an open problem. More specifically, complex constraints and the coupling of transmission time and power on SUs impose enormous challenge on the optimal scheme design in underlay RF-CRNs with residual energy.

\section{System model}

In this paper, we consider an underlay RF-CRN that can work in multi-priority services transmission scenarios, in which a primary network consisting of a primary transmitter (PT) and a primary receiver (PR) and a secondary network consisting of a secondary transmitter (ST) and a secondary receiver (SR) coexist in the same frequency band. It is reasonable to assume that the PU stays in a state of continuous mutual communication under the licensed spectrum as the high priority service demands, so that ST can collect energy from the emitted RF signals of PT to perform data transmission to SR which works in the same band. Therefore, there are three kinds of links in the system, namely, energy link (PT-ST), interference links (PT-SR and ST-PR), and data links (ST-SR and PT-PR), as shown in Fig. 1a. We also assume the licensed channel follows block fading, meaning the fading state between each block is constant, but may vary from one block to another. In order to simplify the model complexity, without loss of generality, we assume that SR has a separate stable power supply, and ST is completely self-powered by the harvested RF energy.

SU works in the energy harvesting-data transmission mode, in which SU first collecting energy and then transmitting data, so two steps need to be completed one after another. As can be seen from Fig. 1b, a data frame with duration $D$ considered for ST can be divided into two time slots, i.e., energy harvesting slot $D-t$ with duration and data transmission slot with duration $t$. And it is assumed that ST is capable of managing the energy, so that the residual energy from previous time slot can be reserved for the next time slot as initial energy. 


\section{a}

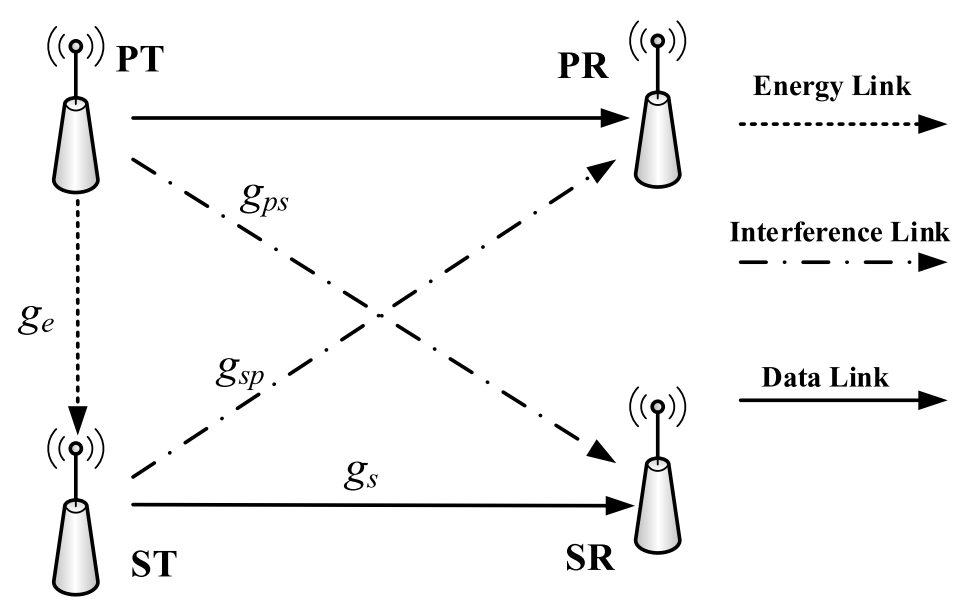

b

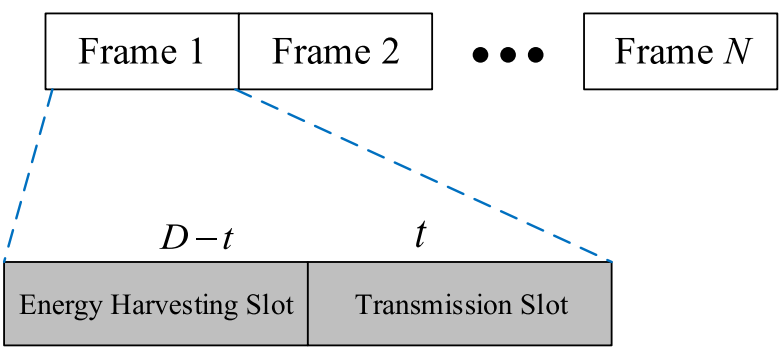

Fig. 1 The model of RF-CRN. a System scenario. b Time slot structure

Energy harvesting slot: Firstly, SU enters the energy harvesting slot, in which the ST collects energy for a duration $D-t$, and the harvested energy, denoted as $E_{\text {harv }}$, can be given by

$$
E_{\text {harv }}=\epsilon g_{e} P_{t}(D-t)
$$

where $\epsilon$ depicts the energy harvesting efficiency of ST, $g_{e}$ denotes the channel gain of energy link from PT to ST and $P_{t}$ is the constant transmission power of PT. It is usually assumed that the energy harvested by ST is kept in an embedded battery with infinite capacity.

Data transmission slot: In data transmission slot, ST transmits information bits to SR with energy stored in the energy storage device. The energy consumed by ST can not exceed the total available energy consisting of harvested energy $E_{\text {harv }}$ and initial energy $Q_{e}$, which is referred to as energy causality constraint [1]

$$
\left(P_{s}+P_{\text {cir }}\right) t \leq E_{\text {harv }}+Q_{e}
$$

where $P_{s}$ and $P_{c i r}$ are the transmission power and the circuit working power consumption of ST, respectively. Since ST shares the same spectrum with PT in underlay mode, the transmission power of ST must be constrained to keep the interference generated from ST to PR within an acceptable threshold, namely interference constraint [1].

$$
P_{s} g_{s p} \leq P_{\text {th }}
$$


where $P_{t h}$ is the peak permissible interference threshold for PR, $g_{s p}$ is the channel gain of the interference link from ST to PR. Then, the achievable throughput of the secondary network can be calculated as follows:

$$
C\left(t, P_{s}\right)=t \log _{2}\left(1+\frac{P_{s} g_{s}}{P_{t} g_{p s}+\sigma^{2}}\right)
$$

where $g_{s}$ is channel gain of the data link from ST to SR and $g_{p s}$ is channel gain of the interference link from PT to SR. We model thermal noise and background interference as additive white Gaussian noise (AWGN), with power spectral density $\sigma^{2}$ received at SR.

To ensure the QoS of secondary network, we impose a minimum throughput requirement $C_{\min }$ on ST.

$$
C\left(t, P_{s}\right) \geq C_{\min }
$$

It is remarkable that the energy harvesting link and interference link are both related to the distance between the primary network and secondary network. In this paper, we consider the small scale fading and the channel gain can be modeled as $g_{X}=\left|G_{X}\right|^{2} S^{-\alpha}(X=$ $e, s p, p s, s)$, where $\alpha$ is the path loss exponent and $S$ is the distance between the users. $G_{X}$ is an exponentially distributed random variable with unit mean [13]. Energy efficiency maximization: During the energy efficiency maximization process, the total energy $E_{t c}\left(t, P_{s}\right)$ consumed by ST consists of two parts: first part is the energy loss due to attenuation of channel propagation in the EH slot and the other part is the energy consumed in data transmission slot. Thus, the total consumed energy $E_{t c}\left(t, P_{s}\right)$ can be calculated as follows:

$$
E_{t c}\left(t, P_{s}\right)=P_{h w}(D-t)+\left(P_{s}+P_{c i r}\right) t
$$

where $P_{h w}(D-t)$ is the amount of RF energy consumed by the PT hardware and cannot be harvested by ST. Combining aforementioned Eqs. (1)-(6), the EE maximization for the underlay RF-CRN is presented as follows:

$$
\begin{aligned}
& \text { OP1 }: \max _{t, P_{s}} E E\left(t, P_{s}\right)=\frac{C\left(t, P_{s}\right)}{E_{t c}\left(t, P_{s}\right)} \\
& \text { s.t. }\left\{\begin{array}{l}
C 1:\left(P_{s}+P_{\text {cir }}\right) t \leq E_{\text {harv }}+Q_{e} \\
C 2: P_{s} g_{s p} \leq P_{t h} \\
C 3: C\left(t, P_{s}\right) \geq C_{\min } \\
C 4: 0<t<D \\
C 5: 0<P_{s}<P_{\max }
\end{array}\right.
\end{aligned}
$$

where constraint $C 1$ means that under the current allocated energy harvesting time and signal transmitting power, the energy consumption of data transmission cannot be higher than the sum of initial energy $Q_{e}$ and energy $E_{\text {harv }}$ harvested by the system; constraint $C 2$ indicates that the transmitting power of ST should not exceed the interference power threshold $P_{t h}$ of PR. In this scenario, we use $C 2$ to protect PR from being interfered by $\mathrm{ST}$ severely. Constraint $\mathrm{C} 3$ is used to restrict the throughput requirement of $\mathrm{SU}$ in this system, which defines the minimum throughput that SU should achieve, and it is the QoS guarantee of the system. Constraint $C 4$ indicates that the total consumed time of SU data transmission cannot be larger than frame period $D$. Finally, $P_{\max }$ represents the maximum transmitting power of ST authorized by the CRN system, while constraint $C 5$ represents the maximum transmitting power of ST that cannot exceed $P_{\max }$. Here, OP1 is a typical nonlinear fractional programming problem, which is difficult to solve in an explicitly way. 


\section{Theoretical analysis and algorithm design}

Obviously, the objective function of $O P 1$ is a non-convex problem and the product of the optimization variables $t$ and $P_{s}$ occurs in constraint $C 1$; hence, $O P 1$ is a non-convex problem. To make $O P 1$ solvable and realizable, a new variable $\eta$, which is defined as $P_{s}=\frac{\eta}{t}$ and can be regarded as the actual energy that ST uses for data transmission, is introduced in this paper. Subsequently, the system model expression $O P 1$ is further transformed into the following form by substituting $\eta$ into (1)-(6) as below

$$
\begin{aligned}
& \text { OP2 }: \max _{t, \eta} E E(t, \eta)=\frac{C(t, \eta)}{E_{t c}(t, \eta)} \\
& \text { s.t. }\left\{\begin{array}{l}
C 1: \eta+P_{\text {cir }} t \leq \epsilon g_{e} P_{t}(D-t)+Q_{e} \\
C 2: \eta g_{s p} \leq t P_{t h} \\
C 3: C(t, \eta) \geq C_{\min } \\
C 4: 0<t<D \\
C 5: 0<\eta<\eta_{\max }
\end{array}\right.
\end{aligned}
$$

where $C(t, \eta)=t \log _{2}\left(1+\frac{\eta}{t} \gamma\right), \gamma=\frac{g_{s}}{P_{\mathrm{t} g} g_{p s}+\sigma^{2}}$ and $E_{t c}(t, \eta)=P_{h w}(D-t)+\eta+$ $P_{\text {cir }}$. $C 1, C 2, C 3$ represent energy constraint, interference constraint, and throughput constraint corresponding to (2), (3), (5), respectively.

Theorem 1 The objective function $E E(t, \eta)$ of problem $O P 2$ is the ratio of a concave function to an affine function.

Proof According to Dinkelbach's basic theory, $C(t, \eta)=t \log _{2}\left(1+\frac{\eta}{t} \gamma\right)$ is the perspective transformation of a function $f(\eta) \triangleq \log _{2}\left(1+\frac{\eta}{t} \gamma\right)$. It is clear that $f(\eta)$ is a concave function of $\eta$ as the logarithmic function is concave. As perspective transformation preserves convexity, so $C(t, \eta)$ is a concave function related to $t$ and $\eta$ [36]. Besides, $E_{t c}(t, \eta)$ is obviously an affine function in terms of $t$ and $\eta$. Therefore, we get Theorem 1 proved.

Therefore, from Theorem 1, the optimization problem OP2 can be converted into a parameterized concave maximization problem by utilizing the well-known Dinkelbach's method [37] as follows:

$$
\begin{aligned}
& \text { OP3 }: \max f(w)=C(t, \eta)-w E_{t c}(t, \eta) \\
& \text { s.t. }\left\{\begin{array}{l}
C 1: \eta+P_{c} t \leq \epsilon g_{e} P_{t}(D-t)+Q_{e} \\
C 2: \eta g_{s p} \leq t P_{t h} \\
C 3: C(t, \eta) \geq C_{\min } \\
C 4: 0<t<D \\
C 5: 0<\eta<\eta_{\max }
\end{array}\right.
\end{aligned}
$$

where $w \in R^{+}$is a weight parameter used in $O P 2$ to optimize $E E(t, \eta)$ values. The maximum value of $E E(t, \eta)$ can be achieved as long as a value of $w^{*}$ that makes $f\left(w^{*}\right)=0$ can be found. In addition, the maximum value of $E E(t, \eta)$ is equal to $w^{*}$ [37]. Evidently, OP3 becomes a convex optimization problem with respect to $t$ and $\eta$ and satisfies Slater's condition, so we can solve its dual problem instead. The partial Lagrangian function of OP3 in term of $C 1$ and $C 3$ can be given as below 


$$
\begin{array}{r}
L(t, \eta, \lambda, \mu) \\
=C(t, \eta)-w E_{t c}(t, \eta)+\mu\left(C(t, \eta)-C_{\min }\right) \\
-\lambda\left(\eta+P_{c i r} t-\epsilon g_{e} P_{t}(D-t)-Q_{e}\right)
\end{array}
$$

where $\lambda$ and $\mu$ are non-negative dual variables associated with $C 1$ and $C 3$, respectively. Then, the Lagrangian dual function of $O P 3$ can be derived as follows

$$
g(\lambda, \mu)=\max _{t, \eta \geq 0} L(t, \eta, \lambda, \mu)
$$

Finally, the dual problem of $O P 3$ can be described as $d(\lambda, \mu)=\min _{\lambda, \mu \geq 0} g(\lambda, \mu)$. As known, the dual function is concave since it is the pointwise infimum of affine functions of $(\lambda, \mu)$, even when the problem is not convex.

Theorem 2 For given $w \geq 0, \lambda \geq 0, \mu \geq 0$, the optimal time and energy allocation can be derived by

$$
t^{*}=\left[-\eta^{*} \gamma \frac{W(\theta)}{W(\theta)+1}\right]^{+}
$$

where $\theta=-\exp \left(-\left(\frac{\zeta \ln 2}{1+\mu}\right)\right), W(\bullet)$ denotes the Lambert $W$ function and $(x)^{+} \triangleq$ $\max (0, x)[38]$. Then, the optimal value of $\eta$ can be obtained as

$$
\eta^{*}=\min \left[t^{*}\left(\frac{1}{\gamma}-\frac{1+\mu}{\ln 2(w+\lambda)}, \frac{t^{*} P_{t h}}{g_{s p}}\right)\right]^{+}
$$

Proof OP3 is a convex optimization problem and the Slater's condition is satisfied. Thus, the optimal $t$ and $\eta$ must satisfy Karush-Kuhn-Tucker (KKT) condition, given as follows"

$$
\begin{aligned}
& \left.\frac{\partial L(t, \eta, \lambda, \mu)}{\partial t}\right|_{t=t^{*}}=0 \\
& \left.\frac{\partial L(t, \eta, \lambda, \mu)}{\partial \eta}\right|_{\eta=\eta^{*}}=0
\end{aligned}
$$

In order to obtain $t^{*}$, we can obtain the results by calculating (14)

$$
\ln \left(1+\frac{\eta^{*}}{t^{*}} \gamma\right)-\frac{\frac{\eta^{*}}{t} \gamma}{1+\frac{\eta^{*}}{t^{*}} \gamma}=\frac{\zeta \ln 2}{1+\mu}
$$

where $\zeta=w P_{h w}+w P_{c}+\lambda P_{c}+\mu g_{e} P_{t}$. Since Lambert W function is the inverse function of $f(z)=z e^{z}$ to solve $t$ [38], we can calculate $t^{*}$ afterwards. Let $x=\frac{\eta}{t} \gamma$ and $\lambda=1+\mu$, we get $A=\frac{\zeta \ln 2}{\lambda}$. Then, we can convert (16) into 


$$
\begin{aligned}
& \ln (1+x)-\frac{x}{1+x}=A \\
& \Rightarrow \ln (1+x) \frac{1}{1+x}=A+1 \\
& \Rightarrow(1+x) \ln (1+x)+1=(A+1)(1+x) \\
& \Rightarrow(1+x)[\ln (1+x)-(A+1)]+1=0 \\
& \Rightarrow(1+x)\left[\ln (1+x)-\ln \left(e^{A+1}\right)\right]+1=0 \\
& \Rightarrow(1+x) \ln \left(\frac{1+x}{e^{A+1}}\right)+1=0 \\
& \Rightarrow \frac{1+x}{e^{A+1}} \ln \left(\frac{1+x}{e^{A+1}}\right)+\frac{1}{e^{A+1}}=0 \\
& \Rightarrow \ln \left(\frac{1+x}{e^{A+1}}\right) \exp \left(\ln \left(\frac{1+x}{e^{A+1}}\right)\right)=-\frac{1}{e^{A+1}} \\
& \Rightarrow \ln \left(\frac{1+x}{e^{A+1}}\right)=W\left(-\frac{1}{e^{A+1}}\right) \\
& \Rightarrow x=e^{A+1} \exp \left(W\left(-\frac{1}{e^{A+1}}\right)\right)-1 \\
& \Rightarrow \frac{\eta \gamma}{t}=e^{A+1} \exp \left(W\left(-\frac{1}{e^{A+1}}\right)\right)-1 \\
& \Rightarrow t=\frac{\eta \gamma}{\epsilon^{A+1} \exp \left(W\left(-e^{-(A+1)}\right)\right)-1}
\end{aligned}
$$

Then, we make use of the Lambert $W$ function which is the inverse relation of the function $\exp (W(x))=\frac{x}{W(x)}$ [38], we have

$$
\begin{aligned}
t & =\frac{\eta \gamma}{e^{A+1} \exp \left(W\left(-e^{-(A+1)}\right)\right)-1} \\
& =\frac{\eta \gamma}{e^{A+1}\left(\frac{\left(-e^{-(A+1)}\right)}{W\left(-e^{-(A+1)}\right)}\right)-1} \\
& =\frac{\eta \gamma}{-\frac{1}{W\left(-e^{-(A+1)}\right)}-1} \\
& =\frac{-\eta \gamma W\left(-e^{-(A+1)}\right)}{W\left(-e^{-(A+1)}\right)+1}
\end{aligned}
$$

Let $\theta=-e^{-(A+1)}=-\exp \left(-\left(\frac{\zeta \ln 2}{1+\mu}+1\right)\right)$, then (12) can be obtained. Finally, as the optimal solution of $t^{*}$ is given by (12), we can get $\eta^{*}$ by calculating (15) as

$$
\eta^{*}=t^{*}\left(\frac{1}{\gamma}-\frac{1+\mu}{\ln 2(w+\lambda)}\right)
$$

As the optimal $\eta^{*}$ also subjects to the interference power constraint $C 2$ of $O P 2$, so the optimal $\eta^{*}$ can be obtained as (13). Unfortunately, as we can see from (12)-(13), $t^{*}$ and $\eta^{*}$ are coupling with each other in each iteration. To circumvent this problem, an iterative process can be introduced as the innermost loop. More specifically, we first calculate $t$ by using (12) with given $\eta$ and then update $\eta$ by using (13) under the condition of $t$ until convergence.

Subsequently, the optimal dual variables $\lambda^{*}$ and $\mu^{*}$ that minimize $g(\lambda, \mu)$ can be achieved by using the sub-gradient method. Specifically, the variables $\lambda$ and $\mu$ can be iteratively updated based on the following iteration procedure until convergence. 


$$
\begin{aligned}
& \lambda^{(k+1)}=\lambda^{(k)}-a \nabla L\left(\lambda^{(k)}\right) \\
& \mu^{(k+1)}=\mu^{(k)}-a \nabla L\left(\mu^{(k)}\right)
\end{aligned}
$$

where $\nabla L\left(\lambda^{(k)}\right)=-\left(\eta^{*}+P_{c} t^{*}-\epsilon g_{e} P_{t}\left(D-t^{*}\right)-Q_{e}\right)$ and $\nabla L\left(\mu^{(k)}\right)=$ $t^{*} \log _{2}\left(1+\frac{\eta^{*}}{t^{*}} \gamma\right)-C_{\min }, a$ denotes the step-size in the $k$ th iteration.

Finally, we update $w$ in the outermost iteration. As long as $f\left(w^{*}\right)=0$ or $\left|f\left(w^{*}\right)\right| \leq \varepsilon$, the optimal resource allocation policy and maximum EE can be achieved. We summarize the solution method as Algorithm 1.

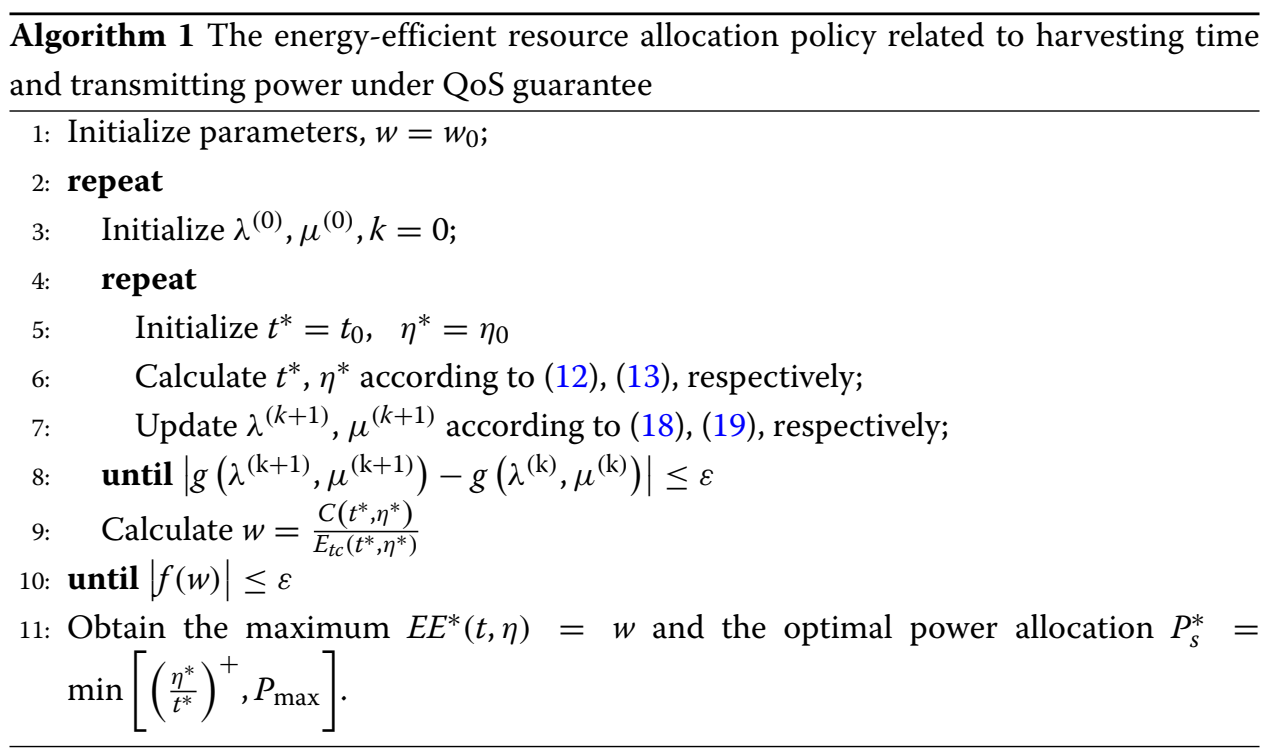

In order to obtain the optimal $w *$ value, the classic Dinkelbach's method is introduced in this paper, which has unique advantages in solving the nonlinear fractional programming problems and has a super-linear convergence rate [37]. Based on the Dinkelbach's method, an optimal resource allocation policy related to transmission time and power is proposed to solve $O P 1$. It is worth noting that since $t$ and $\eta$ following KKT condition are iteratively optimized in the innermost loop, they will converge to the optimal value under the updating process of $\lambda$ and $\mu$. Also, we ensure $\lambda$ and $\mu$ can converge to the optimal solution for given $w$ due to the convexity of OP3 [36]. In summary, our proposed algorithm has a fast convergence rate which consistent with the convergence of Dinkelbach's structure.

According to our analysis, the complexity of algorithm 1 is the computation time of transmission power for Lagrangian dual problem in convex optimization theory. The Lagrangian dual will not change the optimal solution of the original problem but will affect the complexity. For original problem, the complexity of algorithm is related to the sample dimension, while in the Lagrangian dual, the complexity is related to the number of samples, since algorithm 1 is a two-step nested structure of sub-gradient and Lagrangian dual. Therefore, in each iteration of the algorithm, the computational complexity of sub-gradient is $\mathcal{O}(N)$, where $N$ is the number of Lagrangian operators. And the total computational complexity should be $\mathcal{O}\left(N^{2}\right)$. 


\section{Simulation results}

In this section, we present the simulation results to indicate the performance of proposed iterative resource allocation policy updating algorithm. The relative distance between the secondary network and the primary network is about 5 meters. We assume the value of path loss exponent $\alpha$ as 2 [16]. Other scenario parameters are set as follows: normalized frame length $D=1 \mathrm{~s}$, noise power $\sigma^{2}=-65 \mathrm{dBm}$, circuit power consumption $P_{\text {cir }}=$ $0.01 \mathrm{~W}$, PT's transmission power $P_{t} \leq 30 \mathrm{~W}$, interference threshold for PU $P_{t h} \leq 2 \mathrm{~W}$, minimum throughput constraint for SU $C_{\min }=1 \mathrm{bps} / \mathrm{Hz}$ and $\epsilon=0.8$.

Figure 2 demonstrates the convergence rate of the proposed algorithm. Obviously, the proposed algorithm has the feature of fast convergence and insensitivity to the initial values, which conforms to the convergence criterion of the Dinkelbach method [37].

Figure 3a depicts the EE performance versus different primary network settings of $P_{t}$, $P_{t h}$ and minimum throughput requirements of $C_{\min }$. We can see clearly that when $C_{\min }$ is small enough, the maximum EE can be obtained. It means that in the process of EE optimization, ST always sends as few data bits as possible to save energy. When the minimum throughput requirement $C_{\min }$ increases, EE will decrease. Although higher $C_{\min }$ will lead to increases in both throughput and energy consumption, the energy consumption increases even more than the throughput which results in the decline of EE. Besides, EE values stay the same under same values of $P_{t}$ with different $P_{t h}$. Although the expansion of $P_{t h}$ enlarges the range of ST's transmission power $P_{s}, P_{s}$ will stop increasing once it meets the $C_{\min }$. Then, we can see that EE decreases with the increases of $P_{t}$. As larger $P_{t}$ means more interference to the $\mathrm{SR}$, transmission time $t$ and transmission power $P_{s}$ of ST are enlarged in order to satisfy $C_{\min }$ which makes ST consume more energy.

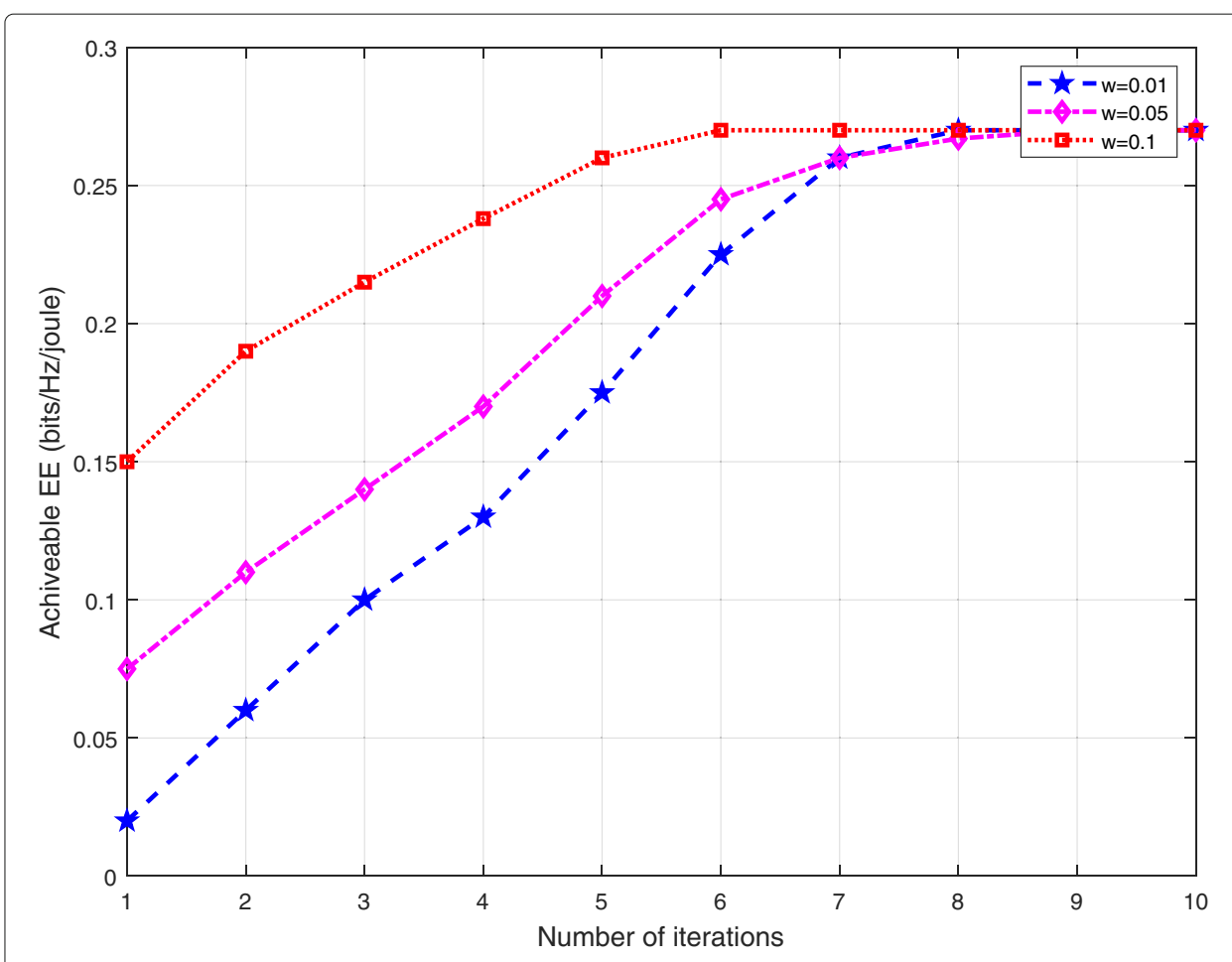

Fig. 2 The convergence analysis result of the proposed algorithm 


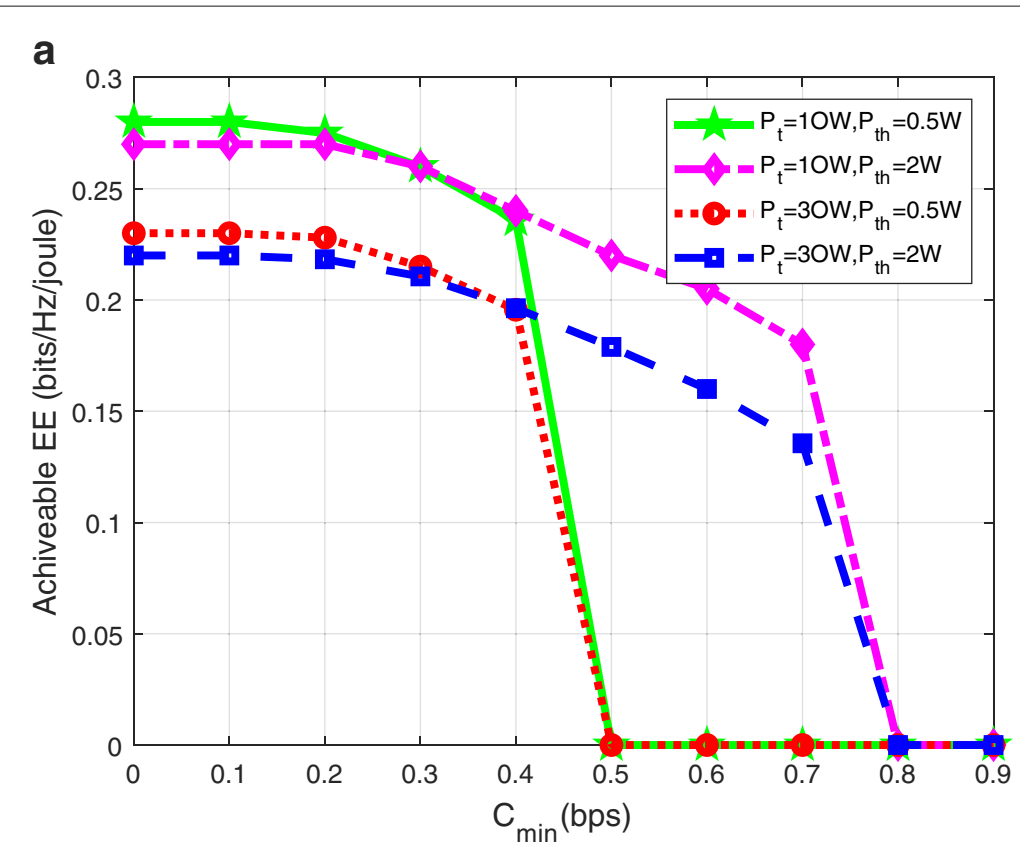

b

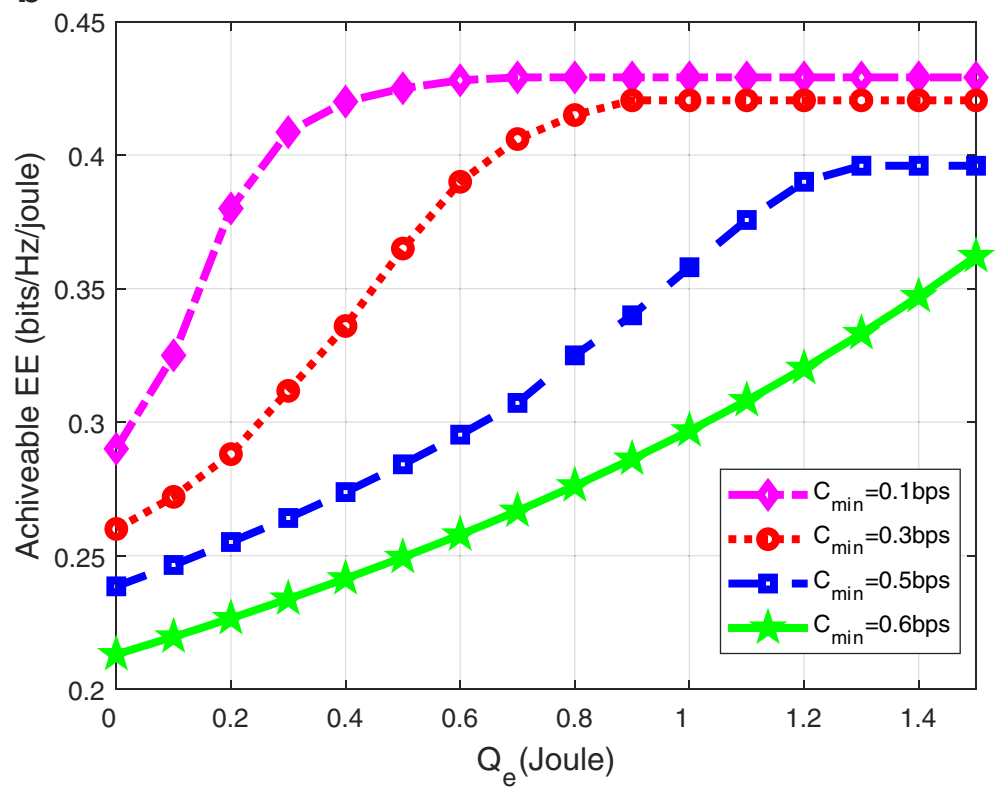

Fig. 3 Comparison of expected EE performance under different $C_{\min }$ and $Q_{e}$ constraints . a Expected EE versus $C_{\min }$ with $Q_{e}=0 . \mathbf{b}$ Expected EE versus $Q_{e}$ with $P_{t}=10 \mathrm{~W}$ and $P_{\text {th }}=2 \mathrm{~W}$

Finally, when the interference is large enough and $P_{t h}$ is tight, then ST cannot satisfy any of the constraints, EE will dramatically decrease to near zero, and the system will be interrupted [39].

Figure $3 \mathrm{~b}$ demonstrates the EE versus different values of initial energy $Q_{e}$. First, when $Q_{e}=0$, ST relies solely on energy harvesting to support the minimum throughput constraint $C_{\min }$ and EE decreases with increasing of $C_{\min }$ which is consistent with the description of Fig. 3a. Subsequently, EE increases with the increasing of $Q_{e}$. The reason 
is that when $Q_{e}$ increases, less time is needed for energy harvesting and more time is allocated to data transmission, thereby the transmission power and the total consumed energy can be reduced. Finally, when $Q_{e}$ continues to increase and can meet the requirement of $C_{\mathrm{min}}$, there is no need to allocate time for ST to harvest energy and the EE will no longer change. It must be noted that, even though it is assumed in the beginning of this paper that the harvested energy of each slot is greater than the energy loss during the entire data block transmission, the $Q_{e}$ size is inversely proportional to the energy harvesting time, and the energy harvesting time is inversely proportional to the transmission time as well, which will not change the influence of $Q_{e}$ during the whole operation process.

Assume $Q_{e}$ stays the same in the following analysis, say, $Q_{e}=0$. Figure 4 shows obviously that the system throughput increases with growth of the $P_{t h}$, due to the fact that the increasing $P_{t h}$ enlarges the feasible domain of ST's transmit power $P_{s}$ and further increases $C\left(t, P_{s}\right)$. Same as EE, $C\left(t, P_{s}\right)$ also decrease with the growth of $P_{t}$. Larger $P_{t}$ means more harvested energy for SU, but also means more interference to SR. Therefore, when interference power is large enough or $P_{t h}$ is tight, the SU cannot satisfy the prescribed minimum throughput threshold, the network throughput dramatically decreases to near zero, and the system interrupted.

Figure $5 \mathrm{a}, \mathrm{b}$ demonstrate that the throughput and energy consumption of the network system when the system achieves the maximum EE under different $C_{\min }$. From Fig. 5, we can observe that $C_{\text {min }}$ has direct impacts on system throughput and energy consumption performance. Although higher $C_{\min }$ will lead to increases in both throughput and energy consumption, the consumption increases more than throughput. Specifically, when $C_{\min }$ is high, more energy is needed to ensure the throughput, which would result in a serious decrease in EE.

Figure 5a illustrates the system throughput versus the minimum rate requirement $C_{\min }$ when the maximum EE is achieved. First, we observe that each expected throughput

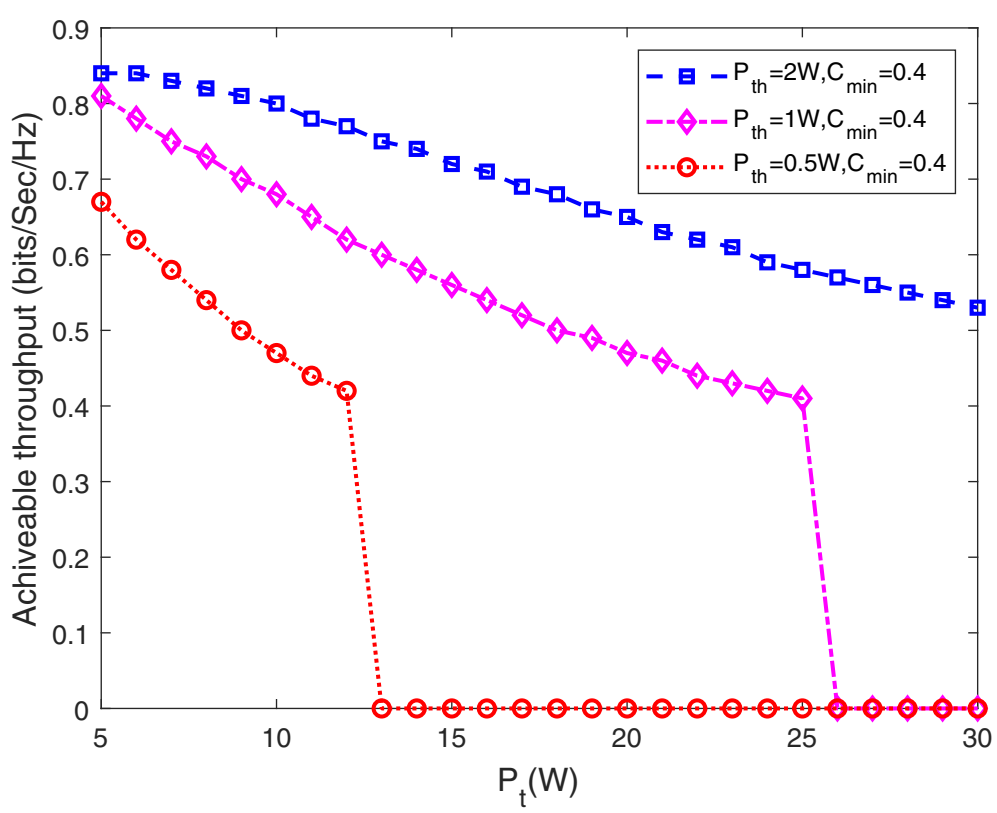

Fig. 4 Achievable throughput optimization performance versus $P_{t}$ 


\section{a}

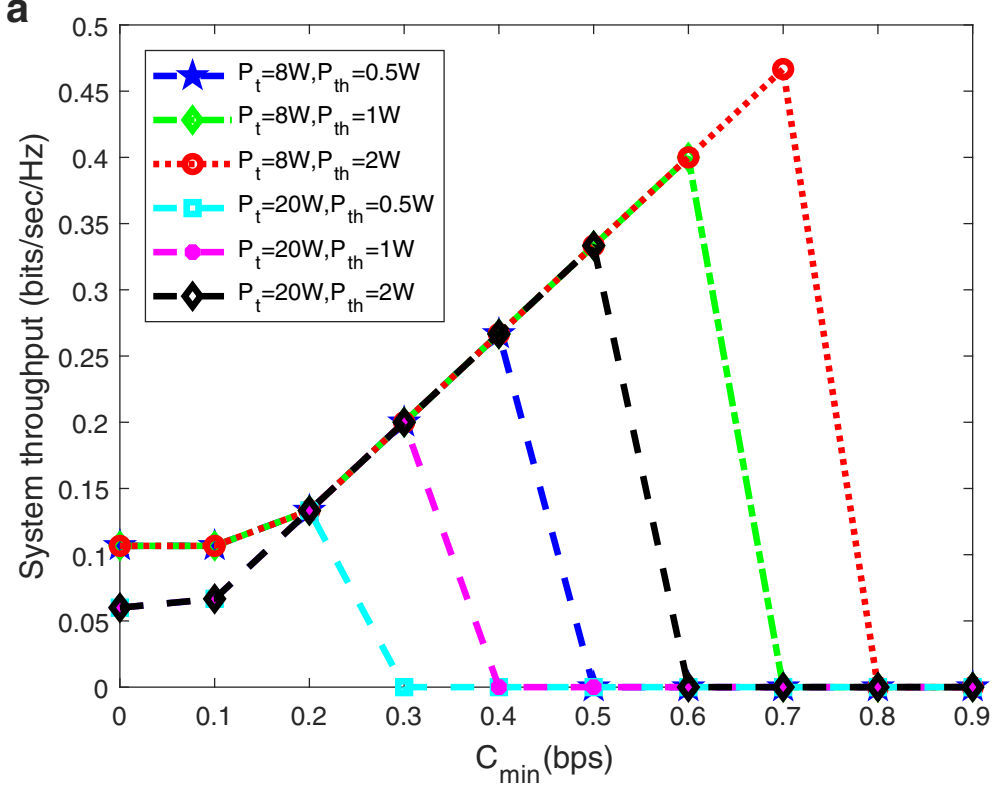

b

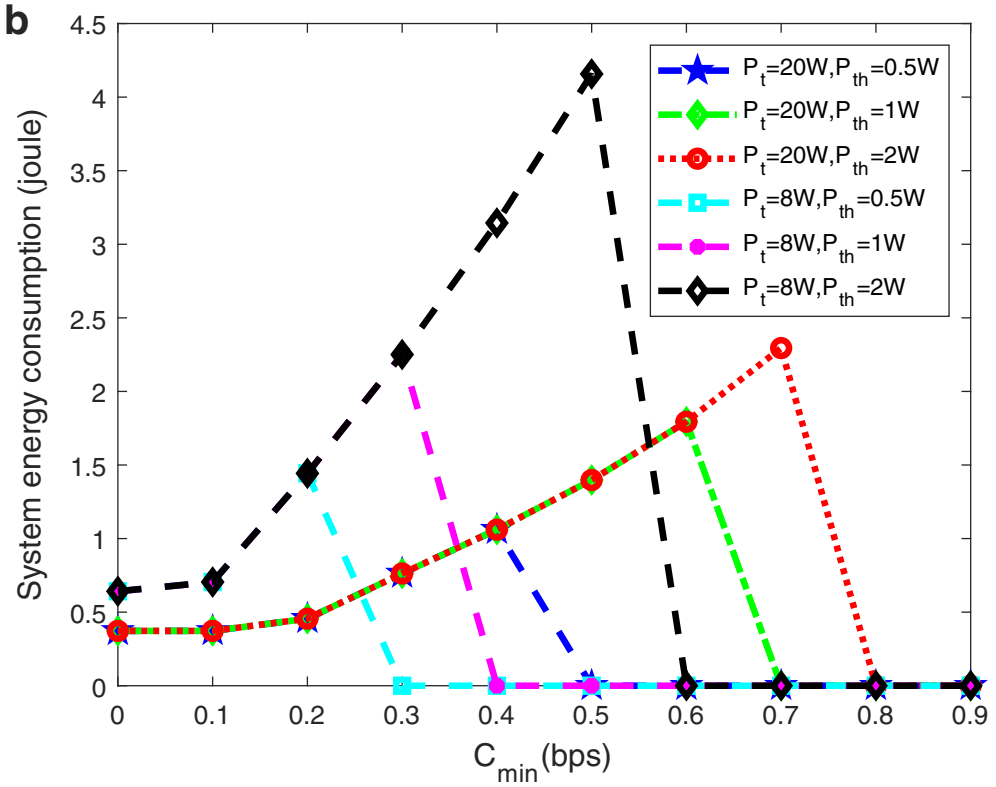

Fig. 5 Expected system performance of throughput/energy consumption versus $C_{\min }$. a System throughput analysis versus $C_{\min }$. b System energy consumption analysis versus $C_{\min }$

curve consists of two parts. The first part is almost fixed, while the second part increases with the minimum rate requirement. Because when the rate requirement is low, very low energy is needed for data transmission, which results in almost fixed throughput. On the other hand, when the rate requirement becomes higher, more energy is needed to transmit data, which results in higher expected throughput. Second, each curve indicates that there exists an upper bound of $C_{\min }$ beyond which the throughput becomes zero due to lack of available solution. Third, in the process of energy efficiency optimization, ST 
always transmits data with same minimum throughput, i.e., $C_{\min }$ under different situations in order to save energy, which leads to the same optimal energy efficiency, which can also be supported by Fig. 4a.

Figure 5b shows the effects of $C_{\min }$ on the expected energy consumption when the maximum $\mathrm{EE}$ is achieved. In general, the energy consumption increases with the increase of $C_{\min }$ and dramatically decreases to near zero when $C_{\min }$ goes beyond the upper bound, due to the fact that the increases of $C_{\min }$ requirement increases throughput and leads to more energy consumption. Obviously, when $C_{\min }$ exceeds an upper value, no available solution exists which means near-zero energy consumption. Lastly, we can observe that larger $P_{t}$ causes more interference to SR leading to the decreasing of throughput, while the transmitting time $\tau$ and $P_{s}$ must be enlarged in order to satisfy the constraint of minimum rate $C_{\min }$ which needs to consume more energy.

In order to guarantee QoS in practical application, it is necessary to select a reasonable throughput threshold; the corresponding constraints should be considered. Because throughput and EE affect each other, a real RF-CRN system must tradeoff between both optimization objectives according to the specific application scenarios. Such as the following:

- For self-powered systems that require long-term operation, like WSNs, we only need to maximize EE based on the basic QoS requirement;

- If the wireless networks need to maximize the total system throughput, then EE optimization should not be treated as the major optimization objective.

\section{Conclusion}

The energy efficiency maximization problem of RF energy harvesting-based cognitive wireless network in underlay mode is modeled and solved in this paper. The throughput threshold is selected to meet the QoS requirements according to different application scenarios and is reflected in the corresponding constraints of the optimization problem. Based on the assumption that ST can reserve the residual energy after previous slot as the initial energy for the next slot, we trade off the performance between energy-efficient transmission and QoS. The optimization algorithm was proposed based on Dinkelbach methods to jointly optimize the energy harvesting time and transmitting power, and the conditions of optimal energy harvesting time and transmitting power were achieved to maximize the energy efficiency of the SU network. The simulation results show that there is a competitive relationship between energy efficiency optimization and throughput requirements. Meanwhile, we found that the setting of PU network scenario and the initial energy of secondary user power supply will have a great impact on the energy efficiency and QoS guarantee of RF-CRN.

\footnotetext{
Abbreviations

EH-CRN: Energy harvesting cognitive radio networks; RF-CRN: Radio frequency energy harvesting-based cognitive radio networks; CRN: Cognitive radio networks; WSN: Wireless sensor networks; RF: radio frequency; PU: Primary user; PT: Primary transmitter; PR: Primary receiver; SU: Secondary user; ST: Secondary transmitter; SR: Secondary receiver; QoS: Quality of service; H-AP: Hybrid access point; WPCN: Wireless powered communication network; WPT: Wireless power transfer; WET: Wireless energy transfer; WIT: Wireless information transmission; SWIPT: Simultaneous wireless information and power transfer DL: Downlink; UL: Uplink; TDM: Time division multiplexing; AP: Access point; CT: Cognitive transmitter
} 


\section{Authors' contributions}

$J T, H X$ and XLL conceived and designed the theory and carried out the theoretical derivation. JT, HX, and XLL participated in the study and performed the simulation experiments, and JT completed the paper writing. JT revised the manuscript and took charge of all the paper submission work. YMS and DH participated in the project and proposed important suggestions on revision. XLL, HX, DH, and YMS reviewed and revised the manuscript. All authors read and approved the final manuscript.

\section{Funding}

The paper is supported by CAEP Foundation under Grant (CX20200010); the General Object of National Natural Science Foundation under Grant (61379005): Research on key technologies for cognitive ad-hoc network based on energy harvesting; the General Object of National Natural Science Foundation under Grant (61771410): Research on decentralized decision-making for cognitive radio ad hoc under uncertainty; and the General Object of National Natural Science Foundation under Grant (61072138): Research on key technologies of intelligent learning and decision-making for cognitive radio.

\section{Availability of data and materials}

Not applicable.

\section{Competing interests}

The authors declare that they have no competing interests.

\section{Author details}

${ }^{1}$ Institute of Electronic Engineering, China Academey of Engineering Physics, No. 64 Mianshan Road, 621900 Mianyang, China. ${ }^{2}$ School of Computer Science, China West Normal University, No. 1 Shida Road, Nanchong, China. ${ }^{3}$ Time and Frequency Research Center, the School of Automation Engineering, University of Electronic Science and Technology of China, No. 2006 Xiyuan Avenue, Chengdu, China.

\section{Received: 30 October 2019 Accepted: 1 October 2020}

Published online: 27 October 2020

\section{References}

1. L. Mohjazi, M. Dianati, G. K. Karagiannidis, S. Muhaidat, M. Al-Qutayri, RF-powered cognitive radio networks: technical challenges and limitations. IEEE Commun. Mag. 53(4), 94-100 (2015)

2. D. Feng, C. Jiang, G. Lim, L. J. Cimini, G. Feng, G. Y. Li, A survey of energy-efficient wireless communications. IEEE Commun. Surv. Tutorials. 15(1), 167-178 (2013)

3. D. T. Hoang, D. Niyato, P. Wang, D. I. Kim, Opportunistic channel access and RF energy harvesting in cognitive radio networks. IEEE J. Sel. Areas Commun. 32(11), 2039-2052 (2014)

4. H. Ju, R. Zhang, Throughput maximization in wireless powered communication networks. IEEE Trans. Wirel. Commun. 13(1), 418-428 (2014)

5. H. Chen, Y. Li, J. L. Rebelatto, B. F. Uch-Filho, B. Vucetic, Harvest-then-cooperate: wireless-powered cooperative communications. IEEE Trans. Sig. Process. 63(7), 1700-1711 (2015)

6. Q. Yao, A. Huang, H. Shan, T. Q. S. Quek, W. Wang, Delay-aware wireless powered communication networks-energy balancing and optimization. IEEE Trans. Wirel. Commun. 15(8), 5272-5286 (2016)

7. Q. Wu, M. Tao, D. W. Kwan Ng, W. Chen, R. Schober, Energy-efficient resource allocation for wireless powered communication networks. IEEE Trans. Wirel. Commun. 15(3), 2312-2327 (2016)

8. X. Lin, L. Huang, C. Guo, P. Zhang, M. Huang, J. Zhang, Energy-efficient resource allocation in TDMS-based wireless powered communication networks. IEEE Commun. Lett. 21(4), 861-864 (2017)

9. M. Song, M. Zheng, Multiuser MISO beamforming for simultaneous wireless information and power transfer. IEEE Trans. Sig. Process. 2(1), 1-4 (2018)

10. J. Xu, L. Liu, R. Zhang, Opportunistic channel access and RF energy harvesting in cognitive radio networks. IEEE J. Sel. Areas Commun. 62(18), 4798-4810 (2014)

11. S. Lee, L. Liu, R. Zhang, Collaborative wireless energy and information transfer in interference channel. IEEE Trans. Wirel. Commun. 14(1), 545-557 (2015)

12. Z. Ding, I. Krikidis, B. Sharif, H. V. Poor, Wireless information and power transfer in cooperative networks with spatially random relays. IEEE Trans. Wirel. Commun. 13(8), 4440-4453 (2014)

13. C. Xu, M. Zheng, W. Liang, H. Yu, Y. Liang, End-to-end throughput maximization for underlay multi-hop cognitive radio networks with RF energy harvesting. IEEE Trans. Wirel. Commun. 16(6), 3561-3572 (2017)

14. S. Yin, Z. Qu, S. Li, Achievable throughput optimization in energy harvesting cognitive radio systems. IEEE J. Sel. Areas Commun. 33(3), 1399-1410 (2016)

15. Y. H. Bae, J. W. Baek, Achievable throughput analysis of opportunistic spectrum access in cognitive radio networks with energy harvesting. IEEE Trans. Commun. 64(4), 3561-3572 (2017)

16. Z. Yang, Z. Ding, P. Fan, G. K. Karagiannidis, Outage performance of cognitive relay networks with wireless information and power transfer. IEEE Trans. Veh. Technol. 65(5), 3828-3833 (2016)

17. Z. Wang, Z. Chen, B. Xia, L. Luo, J. Zhou, Cognitive relay networks with energy harvesting and information transfer: design, analysis, and optimization. IEEE Trans. Wirel. Commun. 15(4), 2562-2576 (2017)

18. V. Rakovic, D. Denkovski, Z. Hadzi-Velkov, L. Gavrilovska, in 2015 IEEE International Conference on Communications (ICC), Optimal time sharing in underlay cognitive radio systems with RF energy harvesting (IEEE, London, 2015), pp. 7689-7694

19. M. Zheng, C. Xu, W. Liang, H. Yu, Harvesting-throughput tradeoff for RF-powered underlay cognitive radio networks. Electron. Lett. 52(10), 881-883 (2016) 
20. Y. Liu, S. A. Mousavifar, Y. Deng, C. Leung, M. Elkashlan, Wireless energy harvesting in a cognitive relay network. IEEE Trans. Wirel. Commun. 15(4), 2498-2508 (2016)

21. C. Wu, Q. Shi, C. He, Y. Chen, Energy utilization efficient frame structure for energy harvesting cognitive radio networks. IEEE Wirel. Commun. Lett. 5(5), 488-491 (2016)

22. F. Zhou, N. C. Beaulieu, Z. Li, J. Si, P. Qi, Energy-efficient optimal power allocation for fading cognitive radio channels: ergodic capacity, outage capacity, and minimum-rate capacity. IEEE Trans. Wirel. Commun. 15(4), 2741-2755 (2017)

23. X. Feng, X. Gan, Energy-efficient design of sensing and transmission in cognitive radio networks. Wirel. Pers. Commun. 84(3), 1647-1662 (2015)

24. X. Liu, J. Yan, in 2016 International Conference on Computing, Networking and Communications (ICNC), Optimal energy-efficient multichannel cognitive radio with primary energy harvesting (IEEE, Kauai, 2016), pp. 1-5

25. M. Robat Mili, L. Musavian, K. A. Hamdi, F. Marvasti, How to increase energy efficiency in cognitive radio networks. IEEE Trans. Commun. 64(5), 1829-1843 (2016)

26. W. Zhang, C. Wang, D. Chen, H. Xiong, Energyspectral efficiency tradeoff in cognitive radio networks. IEEE Trans. Veh. Technol. 65(4), 2208-2218 (2016)

27. L. Zhang, M. Xiao, G. Wu, S. Li, Y. Liang, Energy-efficient cognitive transmission with imperfect spectrum sensing. IEEE J. Sel. Areas Commun. 34(5), 1320-1335 (2016)

28. S. Lee, R. Zhang, Cognitive wireless powered network: spectrum sharing models and throughput maximization. IEEE Trans. Cogn. Commun. Netw. 1(3), 335-346 (2015)

29. D. Xu, Q. Li, Joint power control and time allocation for wireless powered underlay cognitive radio networks. IEEE Wirel. Commun. Lett. 6(3), 294-297 (2017)

30. S. Yin, Z. Qu, S. Li, Achievable throughput optimization in energy harvesting cognitive radio systems. IEEE J. Sel. Areas Commun. 33(3), 407-422 (2015)

31. D. T. Hoang, D. Niyato, P. Wang, D. I. Kim, Performance optimization for cooperative multiuser cognitive radio networks with RF energy harvesting capability. IEEE Trans. Wirel. Commun. 14(7), 3614-3629 (2015)

32. C. Wu, Q. Shi, C. He, Y. Chen, Energy utilization efficient frame structure for energy harvesting cognitive radio networks. IEEE Wirel. Commun. Lett. 5(5), 488-491 (2016)

33. M. Zheng, W. Liang, H. Yu, Harvesting-throughput tradeoff for CDMA-based underlay cognitive radio networks with wireless energy harvesting. IEEE Syst. J. 12(3), 2395-2398 (2018)

34. K. Janghel, S. Prakriya, Throughput of underlay cognitive energy harvesting relay networks with an improved time-switching protocol. IEEE Trans. Cogn. Commun. Netw. 4(1), 66-81 (2018)

35. K. Lee, C. Yoon, O. Jo, W. Lee, Joint optimization of spectrum sensing and transmit power in energy harvesting-based cognitive radio networks. IEEE Access. 6, 30653-30662 (2018)

36. S. P. Boyd, L. Vandenberghe, Convex optimization. (Cambridge University Press, Cambridge, UK;New York, 2004)

37. W. Dinkelbach, On nonlinear fractional programming. Manage. Sci. 13(7), 492-498 (1967)

38. D. A. Barry, J.-Y. Parlange, L. Li, H. Prommer, C. J. Cunningham, F. Stagnitti, Analytical approximations for real values of the Lambert W-function. Math. Comput. Simul. 53(1), 95-103 (2000)

39. X. Zhou, Distributed Real-Time Voltage Regulation in Distribution Networks (2018)

\section{Publisher's Note}

Springer Nature remains neutral with regard to jurisdictional claims in published maps and institutional affiliations.

\section{Submit your manuscript to a SpringerOpen ${ }^{\odot}$ journal and benefit from:}

- Convenient online submission

- Rigorous peer review

Open access: articles freely available online

- High visibility within the field

- Retaining the copyright to your article

Submit your next manuscript at $\gg$ springeropen.com 